\title{
Norepinephrine in the Medial Pre-frontal Cortex Supports Accumbens Shell Responses to a Novel Palatable Food in Food-Restricted Mice Only
}

\author{
Emanuele Claudio Latagliata ${ }^{1}$, Stefano Puglisi-Allegra ${ }^{1,2}$, Rossella Ventura ${ }^{1,2}$ \\ and Simona Cabib ${ }^{1,2 *}$
}

${ }^{1}$ Fondazione Santa Lucia, IRCCS, Rome, Italy, ${ }^{2}$ Daniel Bovet Department of Psychology and Center, Sapienza Università di Roma, Rome, Italy

Previous findings from this laboratory demonstrate: (1) that different classes of addictive drugs require intact norepinephrine (NE) transmission in the medial pre Frontal Cortex $(\mathrm{mpFC})$ to promote conditioned place preference and to increase dopamine (DA) tone in the nucleus accumbens shell (NAc Shell); (2) that only food-restricted mice require intact NE transmission in the mpFC to develop conditioned preference for a context associated with milk chocolate; and (3) that food-restricted mice show a significantly larger increase of mpFC NE outflow then free fed mice when experiencing the palatable food for the first time. In the present study we tested the hypothesis that only the high levels of frontal cortical NE elicited by the natural reward in food restricted mice stimulate mesoaccumbens DA transmission. To this aim we investigated the ability of

OPEN ACCESS

Edited by:

Antonella Gasbarri,

University of L'Aquila, Italy

Reviewed by: Carlos Tomaz,

Universidade Ceuma, Brazil Laura Piccardi,

University of L'Aquila, Italy

*Correspondence: Simona Cabib simona.cabib@uniroma1.it

Received: 09 October 2017 Accepted: 10 January 2018 Published: 26 January 2018

Citation:

Latagliata EC, Puglisi-Allegra S, Ventura $R$ and Cabib $S$, (2018) Norepinephrine in the Medial Pre-frontal Cortex Supports Accumbens Shell Responses to a Novel Palatable Food in

Food-Restricted Mice Only.

Front. Behav. Neurosci. 12:7. doi: 10.3389/fnbeh.2018.00007 a first experience with milk chocolate to increase DA outflow in the accumbens Shell and c-fos expression in striatal and limbic areas of food-restricted and ad-libitum fed mice. Moreover, we tested the effects of a selective depletion of frontal cortical NE on both responses in either feeding group. Only in food-restricted mice milk chocolate induced an increase of DA outflow beyond baseline in the accumbens Shell and a c-fos expression larger than that promoted by a novel inedible object in the nucleus accumbens. Moreover, depletion of frontal cortical NE selectively prevented both the increase of DA outflow and the large expression of c-fos promoted by milk chocolate in the NAc Shell of food-restricted mice. These findings support the conclusion that in food-restricted mice a novel palatable food activates the motivational circuit engaged by addictive drugs and support the development of noradrenergic pharmacology of motivational disturbances.

Keywords: addiction, incentive motivation, novelty response, motivational circuits, salient stimuli, stress

\section{INTRODUCTION}

Dysfunctional processing of motivationally salient stimuli has been proposed as trans-diagnostic phenotype of behavioral disturbances (Robinson and Berridge, 2001; Kapur et al., 2005; Sinha and Jastreboff, 2013; Winton-Brown et al., 2014; Nusslock and Alloy, 2017). Thus, uncovering neurobiological mechanisms of dysfunctional motivation represents a major challenge for basic research. 
Although dopamine (DA) transmission in the Nucleus Accumbens Shell (NAc Shell) plays a paramount role in motivation (Di Chiara and Bassareo, 2007; Cabib and Puglisi-Allegra, 2012; Berridge and Kringelbach, 2015), severe impairment of NAc DA transmission does not always prevent development or expression of motivated responses (Nader et al., 1997). Moreover, pharmacological blockade of DA receptors in the NAc Shell disrupts expression of appetitive/avoidant responses to natural incentives promoted by local antagonism of glutamate receptors, but not those promoted by stimulation of GABAergic transmission (Faure et al., 2008; Richard et al., 2013). Finally, DA and opioids are independently involved in food motivation depending on the organism's state (Bechara and van der Kooy, 1992; Baldo et al., 2013; Fields and Margolis, 2015). These findings support the involvement of different brain circuits in motivation and suggest the hypothesis that dysfunctional motivation could be associated to engagement of a specific brain circuit.

The engagement of NAc in motivational processes is controlled by the medial pre Frontal Cortex (mpFC; Richard and Berridge, 2013; Fiore et al., 2015; Pujara et al., 2016; Quiroz et al., 2016) and frontal cortical norepinephrine (NE) and DA transmission modulate DA release in the NAc Shell in opposite ways. Thus, increased DA transmission in the mpFC constraints mesoaccumbens DA release elicited by stress and novel palatable foods (Deutch et al., 1990; Doherty and Gratton, 1996; Pascucci et al., 2007; Bimpisidis et al., 2013), whereas enhanced NE transmission is responsible for the increase of DA in the NAc Shell promoted by different classes of addictive drugs and by acute stress challenge (Darracq et al., 1998; Ventura et al., 2003, 2005, 2007; Nicniocaill and Gratton, 2007; Pascucci et al., 2007). The observation that mpFC NE-dependent activation of mesoaccumbens DA characterizes the brain response to two known pathogens, i.e., stress and addictive drugs, suggests that engagement of this circuit could increase the risk of dysfunctional motivation. In line with this view, selective depletion mpFC NE prevents both the increase of DA outflow in the NAc and the development of conditioned place preference induced by addictive drugs (Ventura et al., 2003, 2005, 2007).

Enhanced mesoaccumbens DA release promoted by either acute stress challenge (Nicniocaill and Gratton, 2007) or amphetamine administration (Darracq et al., 1998) is selectively prevented by blockade of the low affinity alphal adrenergic receptors that are activated by high concentrations of frontal cortical NE (Ramos and Arnsten, 2007). These findings support the view that both addictive drugs and stress activate mesoaccumbens DA release by promoting a large increase of $\mathrm{NE}$ in mpFC. Recent evidence indicates that food-restricted mice respond to the first experience of a palatable food (milk chocolate) with a significantly larger increase of mpFC NE then ad libitum fed mice. Moreover, although both food restricted and free-fed mice develop conditioned preference for a context paired with milk chocolate, only in the formers this response requires intact frontal cortical NE transmission (Ventura et al., 2008). These findings suggest the hypothesis that in food restricted mice the experience of a novel palatable food engages the motivational circuits typically observed in animals challenged by addictive drugs. To test this hypothesis the following experiments were evaluated: (1) whether milk chocolate elicits an mpFC NE-dependent DA release in the NAc Shell of food-restricted mice; and (2) whether the first experience of milk chocolate promotes a different pattern of c-fos expression in limbic and striatal brain regions of ad libitum fed and food-restricted mice.

\section{MATERIALS AND METHODS}

\section{Animals and Housing}

Male mice of the inbred C57BL/6JIco strain (Charles River, Como, Italy), 8-9 weeks old at the time of the experiments, were housed as previously described and maintained in a $12 \mathrm{~h} / 12 \mathrm{~h}$ light/dark cycle (light on between 07.00 a.m. and 07.00 p.m.). Each experimental group consisted of 5-8 animals. All animals were treated in accordance with the principles expressed in the Declaration of Helsinki. All experiments were carried out in accordance with Italian national law (DL 116/92 and DL 26/2014) on the use of animals for research based on the European Communities Council Directives (86/609/EEC and 2010/63/UE), and approved by the ethics committee of the Italian Ministry of Health (license/approval ID \#: 10/2011-B and 42/2015-PR).

Mice were individually housed and assigned to different feeding regimen, namely either receiving food ad libitum (FF) or subjected to food-restriction regimen (FR). FR mice received food once daily (07.00 p.m.) in a quantity adjusted to induce a loss of $15 \%$ of the original body weight. In the FF condition, food was given once daily (07.00 p.m.) in a quantity adjusted to exceed daily consumption (17 g; Ventura and Puglisi-Allegra, 2005; Ventura et al., 2008). Differential feeding regimen started 4 days before experiments.

\section{Drugs}

Zoletil 100, Virbac, Milano, Italy (tiletamine $\mathrm{HCl} 50 \mathrm{mg} / \mathrm{ml}$ + zolazepam $\mathrm{HCl} 50 \mathrm{mg} / \mathrm{ml}$ ) and Rompun 20, Bayer S.p.A Milano, Italy (xylazine $20 \mathrm{mg} / \mathrm{ml}$ ), purchased commercially, were used as anesthetics, 6-hydroxydopamine (6-OHDA) and GBR 12909 (GBR), were purchased from Sigma (Sigma Aldrich, Milan, Italy). Zoletil (30 mg/kg), Rompun (12 mg/kg) and GBR $(15 \mathrm{mg} / \mathrm{Kg})$ were dissolved in saline $(0.9 \% \mathrm{NaCl})$ and injected intraperitoneally (i.p.) in a volume of $10 \mathrm{ml} / \mathrm{kg}$. 6-OHDA was dissolved in saline containing Na-metabisulfite (0.1 M).

\section{Stimuli}

A piece of milk chocolate ( $1 \mathrm{~g}$, Milka $\odot$ : Fat $=29.5 \%$; Carbs $58.5 \%$; Proteins 6.6\%) was used as palatable food in all experiments (MC). A piece of Lego $\odot$ of the same size was used to control for stimulus novelty in the fos experiments and in conditioned place preference (CPP; OBJ). FF mice consumed $0.1 \pm 0.05 \mathrm{~g}$ of MC and FR mice $0.7 \pm 0.1$ ( $p<0.01, t$-test $)$ in the $40 \mathrm{~min}$ of exposure, regardless of experimental condition.

\section{NE Depletion in the mpFC}

Animals were anesthetized with Zoletil and Rompun, then mounted in a stereotaxic frame (David Kopf Instruments, Tujunga, CA, USA) equipped with a mouse adapter. Mice 
were injected with $\mathrm{GBR}(15 \mathrm{mg} / \mathrm{Kg}$, ip) $30 \mathrm{~min}$ before the 6-OHDA micro-injection in order to protect dopaminergic neurons. Bilateral injection of 6-OHDA $(1.5 \mu \mathrm{g} / 0.1 \mathrm{ml} / 2 \mathrm{~min}$ for each side) was made into the mpFC (coordinates: +2.52 AP; $\pm 0.6 \mathrm{~L} ;-2.0 \mathrm{~V}$ with respect to bregma (Franklin and Paxinos, 2001), through a stainless steel cannula $(0.15 \mathrm{~mm}$ outer diameter, UNIMED, Switzerland), connected to a $1 \mu$ l syringe by a polyethylene tube and driven by a CMA/100 pump (NE depleted group). The cannula was left in place for an additional $2 \mathrm{~min}$ after the end of the infusion. Sham animals were subjected to the same treatment, but received intracerebral vehicle. Note that in previous experiments we observed no significant difference between Sham-treated and naïve animals in basal or pharmacological/natural stimuli-induced prefrontal NE or DA outflow or in CPP or conditioned place aversion (CPA) test (Ventura et al., 2005, 2007; Pascucci et al., 2007), thus ruling out the action of GBR on observed effects in present experiments.

In all experiments animals were used 7 days after surgery.

$\mathrm{NE}$ and DA tissue levels in the mpFC were assessed, as previously described (Ventura et al., 2003, 2005, 2007), to evaluate the extent of depletion. In the microdialysis experiments mice were killed by decapitation to collect tissue samples from mpFC when DA levels in the NAc Shell returned to baseline (120 min after the first sampling). In the case of c-fos experiments, the frontal pole was excised immediately before the brain immersion in formalin (see "Immunostaining and Image Analyses" section). Finally, two groups (sham-depleted and NEdepleted) of unhandled mice were sacrificed 10 days after surgery to evaluate NE and DA tissue levels in both the mpFC and NAc Shell. The latter group of mice was added to rule out a subcortical spill over of the neurotoxin.

\section{Microdialysis}

Anesthesia and surgical set are the same as described for NE depletion. Mice were implanted unilaterally with a guide cannula (stainless steel, shaft OD $0.38 \mathrm{~mm}$, Metalant AB, Stockholm, Sweden) in the NAc Shell (Ventura et al., 2003, 2005, 2007). The $4.5 \mathrm{~mm}$-long guide cannula was fixed with epoxy glue; dental cement was added for greater stability. The coordinates from bregma (measured according to Franklin and Paxinos, 2001) were: +1.60 anteroposterior and 0.6 lateral. The probe (dialysis membrane length $1 \mathrm{~mm}$, o.d. $0.24 \mathrm{~mm}$, MAB 4 cuprophane microdialysis probe, Metalant $A B$ ) was introduced $24 \mathrm{~h}$ before microdialysis experiments. Animals were lightly anesthetized to facilitate manual insertion of the microdialysis probe into the guide cannula and were then returned to their home cages. The outlet and inlet probe tubing was protected by locally applied parafilm. The membranes were tested for in vitro recovery of DA (relative recovery $(\%): 10.7 \pm 0.82 \%)$ on the day before use in order to verify recovery.

The microdialysis probe was connected to a CMA/100 pump (Carnegie Medicine Stockholm, Sweden) through PE-20 tubing and an ultra-low torque dual channel liquid swivel (Model 375/D/ 22QM, Instech Laboratories, Inc., Plymouth Meeting, PA, USA) to allow free movement. Artificial CSF (147 mM
$\mathrm{NaCl}, 1 \mathrm{mM} \mathrm{MgCl}, 1.2 \mathrm{mM} \mathrm{CaCl}_{2}$ and $4 \mathrm{mM} \mathrm{KCl}$ ) was pumped through the dialysis probe at a constant flow rate of $2 \mu \mathrm{l} / \mathrm{min}$. Experiments were carried out $22-24 \mathrm{~h}$ after probe placement. Each animal was placed in a circular cage furnished with microdialysis equipment (Instech Laboratories, Inc.) and with home cage bedding on the floor. Dialysis perfusion was started $1 \mathrm{~h}$ later, at which time the mice were left undisturbed for approximately $2 \mathrm{~h}$ before baseline samples were collected. The mean concentration of the three samples collected immediately before testing (less than 10\% variation) was taken as basal concentration.

Immediately after collection of the three baseline samples the piece of chocolate (MC) was introduced to the cage. Dialysate was collected twice over a $40 \mathrm{~min}$ test to keep the experience within the time limit of a CPP training session. Only data from mice with a correctly placed cannula are reported. Placements were judged by methylene blue staining. Twenty microliters of the dialysate samples were analyzed by high performance liquid chromatography (HPLC). The remaining $20 \mu \mathrm{l}$ were kept for possible subsequent analysis. Concentrations $(\mathrm{pg} / 20 \mu \mathrm{l})$ were not corrected for probe recovery. The HPLC system consisted of an Alliance (Waters Corporation, Milford, MA, USA) system and a coulometric detector (ESA Model 5200A Coulochem II) provided with a conditioning cell (M 5021) and an analytical cell (M 5011). The conditioning cell was set at $400 \mathrm{mV}$, electrode 1 at $200 \mathrm{mV}$ and electrode 2 at $-150 \mathrm{mV}$. A Nova-Pack C18 column $(3.9 \times 150 \mathrm{~mm}$, Waters $)$ maintained at $30^{\circ} \mathrm{C}$ was used. The flow rate was $1.1 \mathrm{ml} / \mathrm{min}$. The mobile phase was as previously described (Ventura et al., 2007, 2008). The assay detection limit was $0.1 \mathrm{pg}$.

\section{Immunostaining and Image Analyses}

FF and FR mice, either Sham or NE-depleted, were individually exposed to an empty cage, similar to the home-cage but without food or water, $1 \mathrm{~h}$ daily for four consecutive days to reduce $\mathrm{c}$-fos activation promoted by novel environment. On the 5 th day a novel stimulus (MC or OBJ, see "Stimuli" section for details) was placed in the test cage before the mouse. Mice were left with the stimulus for $40 \mathrm{~min}$, to match duration of training sessions in CPP and of dialysate collection, then were removed and left in their home cages for the following 20 min before killing by decapitation. This procedure was adopted because of previous and preliminary data indicating that in mice $60 \mathrm{~min}$ are required for induced accumulation of c-fos proteins (Conversi et al., 2006; Colelli et al., 2010, 2014).

Following removal of the frontal pole, to be used for evaluation of NE depletion, brains were immersed in chilled $10 \%$ neutral buffered formalin and stored overnight and then cryoprotected in $30 \%$ sucrose solution at $4^{\circ} \mathrm{C}$ for $48 \mathrm{~h}$ (Conversi et al., 2004; Paolone et al., 2007; Colelli et al., 2010, 2014). Frozen coronal sections (40 $\mu \mathrm{m}$ thickness) were cut through whole brain with a sliding microtome and then immunolabeled with immunoperoxidase method as previously described (Conversi et al., 2006; Colelli et al., 2010, 2014). Rabbit anti- c-fos (1/20,000; Oncogene Sciences) was used as primary antibody and secondary immunodetection was performed with a biotinylated antibody (1:1000 goat 
anti-rabbit, Vector Laboratories Inc., Burlingame, CA, USA). Peroxidase labeling was obtained by standard avidin-biotin procedure (Vectastain ABC elite kit, Vector Laboratories, diluted 1:500) and chromogenic reaction was developed by incubating sections with metal-enhanced DAB (Vector Laboratories). Immunohistochemical analyses of tissue samples obtained from FF and FR mice were performed in different batches.

Sections were analyzed using a Nikon Eclipse 80i microscope equipped with a Nikon DS-5M CCD camera as previously described (Conversi et al., 2006; Colelli et al., 2010, 2014). Specimens were subjected to quantitative image analysis using the public domain image analysis software IMAGEJ $1.38 \mathrm{~g}$ for Linux (Abramoff et al., 2004). Immunoreactive nuclei density was measured and expressed as number of nuclei/ $0.1 \mathrm{~mm}^{2}$.

\section{Place Conditioning}

Behavioral experiments were performed using a place conditioning apparatus (Cabib et al., 2000; Ventura et al., 2003, 2008). The apparatus comprised two gray Plexiglas chambers $(15.6 \times 15.6 \times 20 \mathrm{~cm})$ and a central alley $(15.6 \times 5.6 \times 20 \mathrm{~cm})$. Two sliding doors $(4.6 \times 20 \mathrm{~cm})$ connected the alley to the chambers. In each chamber two triangular parallelepipeds $(5.6 \times 5.6 \times 20 \mathrm{~cm})$ made of black Plexiglas and arranged in different patterns (always covering the surface of the chamber) were used as conditioned stimuli. The training procedure for place conditioning has been described previously (Cabib et al., 2000; Ventura et al., 2003, 2008). Briefly, on day 1 (pretest), mice were free to explore the entire apparatus for $20 \mathrm{~min}$. During the following 8 days (conditioning phase) mice were confined daily for $40 \mathrm{~min}$ alternately in one of the two chambers. For half of the animals (from FR and FF groups) one pattern was consistently paired with MC (1 g) and the other with standard food (mouse standard diet $1 \mathrm{~g}$ ); for the other half one pattern was consistently paired with $\mathrm{MC}(1 \mathrm{~g})$ and the other with OBJ.

\section{Statistics}

Four groups of mice were used for the microdialysis experiment: FF sham, $n=7$; FF depleted, $n=5$; FR sham, $n=6$; FR depleted, $n=6$. Data (DA output: pg/20 $\mu \mathrm{l}$ ) were analyzed by two-way ANOVAs with a within factor (minute blocks following exposure to MC) and an independent factor: treatment (6-OHDA depletion or Sham depletion). The simple effect of the repeated measure (time-dependent variation of the DA levels) was also evaluated within each group.

Six groups of mice were used for the fos experiments ( $n=5$ each). Data (density of c-fos immunostained nuclei) were analyzed by two-way ANOVAs with two independent variables: novel stimulus (MC or OBJ) and treatment (6-OHDA depletion or Sham depletion). Post hoc analyses (Tukey's correction) were performed whenever a significant interaction between factors was revealed.

Four groups of mice were used for the CPP experiments: 1 group of FF and 1 group of FR mice ( $n=8$ each) was trained to discriminate a compartment paired with $\mathrm{MC}$ and one paired with standard food chow and another group of FF $(n=8)$ and of FR $(n=7)$ mice was trained to discriminate a compartment paired with $\mathrm{MC}$ and one paired with an inedible object. Behavioral data (seconds spent in compartment) were analyzed by two-way ANOVAs with a within factor (compartment) and an independent factor (feeding state: FF, FR). The simple within-group effect of the compartment was evaluated within each group when a significant interaction between factors was revealed.

\section{RESULTS}

\section{Effects of 6-OHDA Infusion in the mpFC on Tissue Catecholamines Content}

Tissue levels of DA and NE in Sham and NE-depleted mice from the different experiments are reported in Table 1. In all cases, local 6-OHDA infusion under GBR protection significantly reduced NE but did not affect DA levels mpFC. Levels of NE and DA in the NAc Shell were also evaluated in separate groups of mice (Unhandled) to test diffusion of the neurotoxin in this brain area. The results indicate no effects of mpFC NE depletion on either DA or NE in the NAc Shell.

\section{Experiment 1: DA Outflow in the NAc Shell of Mice Exposed to MC for the First Time}

The effects of 40 min of experience with MC on DA outflow in the NAc Shell are reported in Figure 1. Statistical analysis of data collected in FF mice did not reveal any main effect or significant interaction between factors; indeed, neither exposure to $\mathrm{MC}$ nor mpFC NE depletion influenced DA outflow in the NAc

TABLE 1 | Tissue levels of norepinephrine (NE) and dopamine (DA) in Sham and 6OHDA-treated mice.

\begin{tabular}{|c|c|c|}
\hline & Free fed & Food restricted \\
\hline & \multicolumn{2}{|c|}{ Norepinephrine (ng/g wet weight \pm SE) } \\
\hline & \multicolumn{2}{|c|}{ Microdialysis (mpFC) } \\
\hline Sham & $647.5 \pm 28.6(7)$ & $639.6 \pm 37.6(6)$ \\
\hline \multirow[t]{2}{*}{ 6-OHDA } & $45.2 \pm 31.1(5)^{*}$ & $34.8 \pm 9.0(6)^{*}$ \\
\hline & \multicolumn{2}{|c|}{ Immunohistochemistry (mpFC) } \\
\hline Sham & $665.5 \pm 28.0(7)$ & $658.4 \pm 45.1(7)$ \\
\hline \multirow[t]{2}{*}{$6-\mathrm{OHDA}$} & $57.2 \pm 16.5(7)^{*}$ & $47.7 \pm 21.9(7)^{*}$ \\
\hline & \multicolumn{2}{|c|}{ Unhandled Groups (mpFC) } \\
\hline Sham & $672.2 \pm 36.6(8)$ & $657.2 \pm 22.4(8)$ \\
\hline \multirow[t]{2}{*}{$6-\mathrm{OHDA}$} & $68.9 \pm 24.3(8)^{*}$ & $56.0 \pm 32.1(8)^{*}$ \\
\hline & \multicolumn{2}{|c|}{ Unhandled Groups (NAc Shell) } \\
\hline Sham & $743.6 \pm 42.1(8)$ & $750.7 \pm 44.6(8)$ \\
\hline \multirow[t]{3}{*}{$6-\mathrm{OHDA}$} & $736.0 \pm 51.7(8)$ & $764.6 \pm 34.2(8)$ \\
\hline & \multicolumn{2}{|c|}{ Dopamine (ng/g wet weight \pm SE) } \\
\hline & \multicolumn{2}{|c|}{ Microdialysis (mpFC) } \\
\hline Sham & $181.6 \pm 15.3(7)$ & $202.8 \pm 21.0(6)$ \\
\hline \multirow[t]{2}{*}{$6-\mathrm{OHDA}$} & $193.6 \pm 29(5)$ & $168.4 \pm 25.8(6)$ \\
\hline & \multicolumn{2}{|c|}{ Immunohistochemistry (mpFC) } \\
\hline Sham & $197.1 \pm 16.1(7)$ & $187.5 \pm 18.5(7)$ \\
\hline \multirow[t]{2}{*}{$6-\mathrm{OHDA}$} & $187.1 \pm 28.1(7)$ & $202.1 \pm 18.6(7)$ \\
\hline & \multicolumn{2}{|c|}{ Unhandled Groups (mpFC) } \\
\hline Sham & $191.2 \pm 29.8(8)$ & $195.2 \pm 16.3(8)$ \\
\hline \multirow[t]{2}{*}{$6-\mathrm{OHDA}$} & $166.2 \pm 19.5(8)$ & $159.1 \pm 22.0(8)$ \\
\hline & \multicolumn{2}{|c|}{ Unhandled Groups (NAc Shell) } \\
\hline Sham & $12646.8 \pm 438.4(8)$ & $12876.2 \pm 418.1(8)$ \\
\hline $6-\mathrm{OHDA}$ & $12344.8 \pm 370.1(8)$ & $12511.7 \pm 407.1(8)$ \\
\hline
\end{tabular}

Note: $* p<0.0001:$ t-test, 2-tailed 


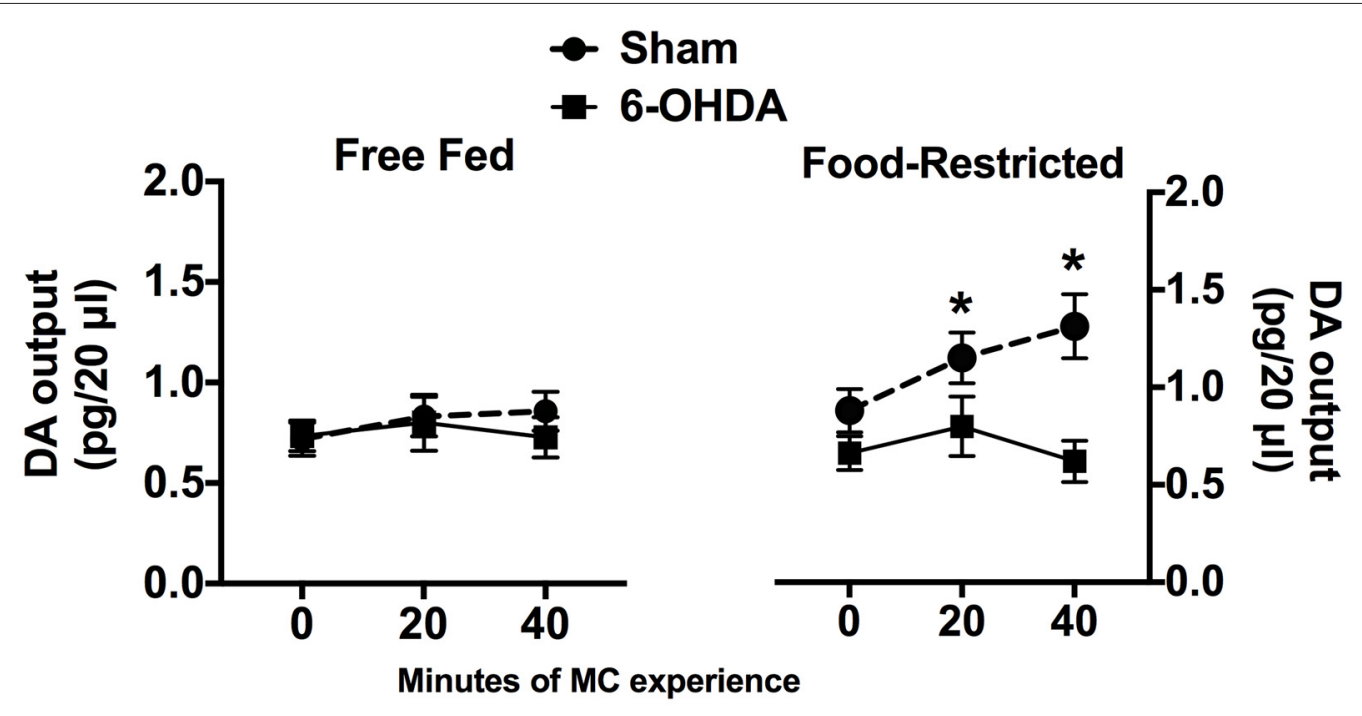

FIGURE 1 | Effects of selective medial pre Frontal cortex (mpFC) norepinephrine (NE) depletion on dopamine (DA) outflow (mean pg/ $20 \mu \mathrm{l} \pm \mathrm{SEM}$ ) in the nucleus accumbens shell (NAc Shell) of Free fed (FF) and Food-restricted (FR) mice. *Significantly different $(p<0.05)$ from concentration at baseline $(0)$.

Shell (Figure 1, left). Instead, a significant interaction between factors was revealed for data collected in FR mice $\left(F_{(2,20)}=11.19\right.$; $p<0.001)$, due to a progressive increase of DA outflow in comparison with baseline $(0)$ in sham-operated animals that was abolished by mpFC NE depletion (Figure 1, right).

\section{Experiment 2: C-fos Immunostaining in Mice Exposed to MC or to an Inedible Object for the First Time}

The effects of $40 \mathrm{~min}$ exposure to $\mathrm{MC}$ or to $\mathrm{OBJ}$ on c-fos expression are shown in Figure 2. Representative images of NAc c-fos expression in the different experimental groups are shown in Figure 3. It should be pointed out that, due to the high number of tissue samples used in these experiments, the samples collected in FF and FR mice were processed in different batches, therefore direct comparison between results obtained in these two groups is not meaningful.

Statistical analyses performed on data collected in FF mice revealed a significant main effect of the factor stimulus (MC vs. $\mathrm{OBJ})$ in the Central Amygdala $\left(\mathrm{CeA} ; F_{(1,28)}=7.35 ; p<0.05\right)$, due to higher $\mathrm{c}$-fos expression in mice exposed to $\mathrm{MC}$ regardless of the treatment (Figure 2, bottom left), and in the Dorsomedial Striatum $\left(\mathrm{DMS} ; F_{(1,28)}=14.44 ; p<0.001\right)$ due to higher $\mathrm{c}$-fos expression in mice exposed to OBJ regardless of the treatment (Figure 2, top left). No effect of NE depletion nor significant interaction between factors stimulus and treatment were revealed by the statistical analyses of data collected in FF mice, indicating that mpFC NE depletion was totally ineffective in FF mice.

As for data collected in FR mice (Figure 2, right) statistical analyses revealed significant interactions between the factors stimulus (OBJ vs. $\mathrm{MC}$ ) and treatment (Sham vs. NE-depleted) in the DMS $\left(F_{(1,24)}=11.5 ; p<0.005\right)$, NAc Core $\left(F_{(1,24)}=12.28\right.$; $p<0.005)$, and NAc Shell $\left(F_{(1,24)}=16.28 ; p<0.001\right)$.
In sham-operated mice $\mathrm{MC}$ promoted a larger increase of c-fos immunostained nuclei then OBJ in NAc Core and Shell (Figure 2, right). This effect was not observed in NE-depleted animal due to a decrease of $\mathrm{MC}$-induced $\mathrm{c}$-fos expression in the NAc Shell and an increase of OBJ-induced c-fos expression in the NAc Core. In the DMS of sham-operated FR mice OBJ was unable to promote $\mathrm{c}$-fos expression higher than that promoted by MC (Figure 2, top right). Frontal cortical NE depletion significantly increased c-fos expression promoted by $\mathrm{OBJ}$ in the DMS, thus recovery the pattern of c-fos activation observed in FF mice.

In the $\mathrm{CeA}$ of FR mice statistical analyses only revealed a main effect of the factor stimulus (MC vs. OBJ; $F_{(1,24)}=24.93$; $p<0.0001)$ due to higher $c$-fos expression in mice exposed to $\mathrm{MC}$ regardless of the treatment (Figure 2, bottom right).

\section{Experiment 3: Conditioned Preference for MC-paired Context}

In Figure 4 are reported data from the CPP experiments. Either FR or FF mice showed significant preference for the compartment paired with MC when the other was paired with the habitual chow food (main effect of the pairing regardless of the feeding condition $F_{(1,13)}=12.36$; $p<0.005$; Figure 4A). Instead, when the other compartment was paired with $\mathrm{OBJ}$ (Figure 4B), only FR mice showed a significant preference for the MC-paired one (significant interaction between pairing and feeding condition: $\left.F_{(1,13)}=5.382 ; p<0.05\right)$.

\section{DISCUSSION}

Major findings of the present study are: (1) only sham-treated FR mice showed increased DA outflow in the NAc Shell during the first experience with MC; (2) only sham-treated FR mice showed 


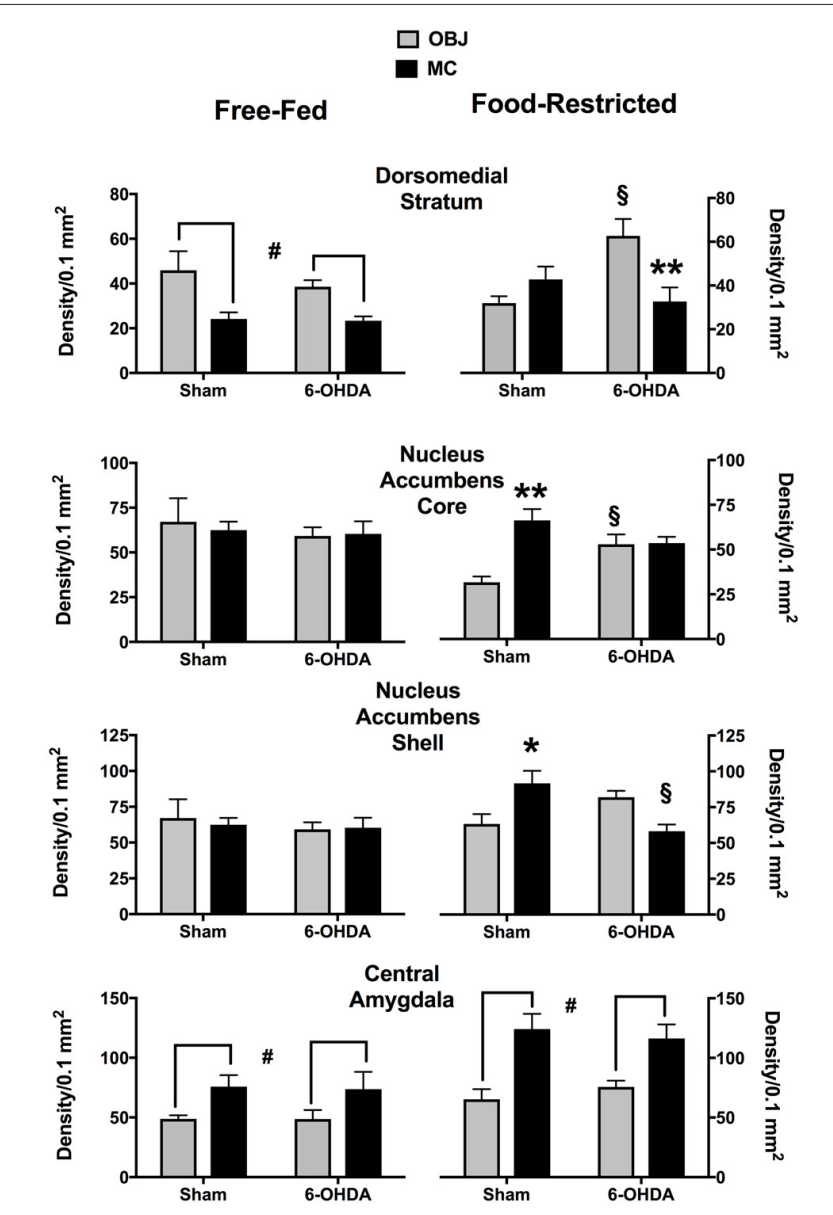

FIGURE 2 | C-fos expression (mean density \pm SEM) induced by the first exploration of a small piece of plastic (OBJ) or a piece of milk chocolate (MC) in different experimental conditions. "Main effect of the novel stimulus (OBJ vs. MC; see text for details). ${ }^{* * * *} p<0.05, p<0.01 \mathrm{vs.} \mathrm{OBJ} \mathrm{(Tukey} \mathrm{correction} \mathrm{for}$ multiple comparisons). ${ }^{\S} p<0.05$ vs. Sham (Tukey correction for multiple comparisons).

MC-induced c-fos expression in the NAc Shell larger than that elicited by a novel inedible object; (3) in the DMS of FF mice and in mpFC NE-depleted FR mice a novel inedible object promoted c-fos expression larger than that promoted by the palatable food; and (4) although both FF and FR mice developed conditioned preference for MC-paired context when the other was associated with habitual food, only FR mice developed preference for the compartment paired with the palatable food when the other was associated with object novelty.

\section{Food Restricted but Not ad libitum Fed Mice Show Enhanced DA Outflow in the NAc Shell When Experiencing Milk Chocolate for the First Time and This Response Is Prevented by Depletion of Frontal Cortical NE}

A first set of experiments demonstrated that initial experience with MC promotes an increase of DA outflow in the NAc Shell

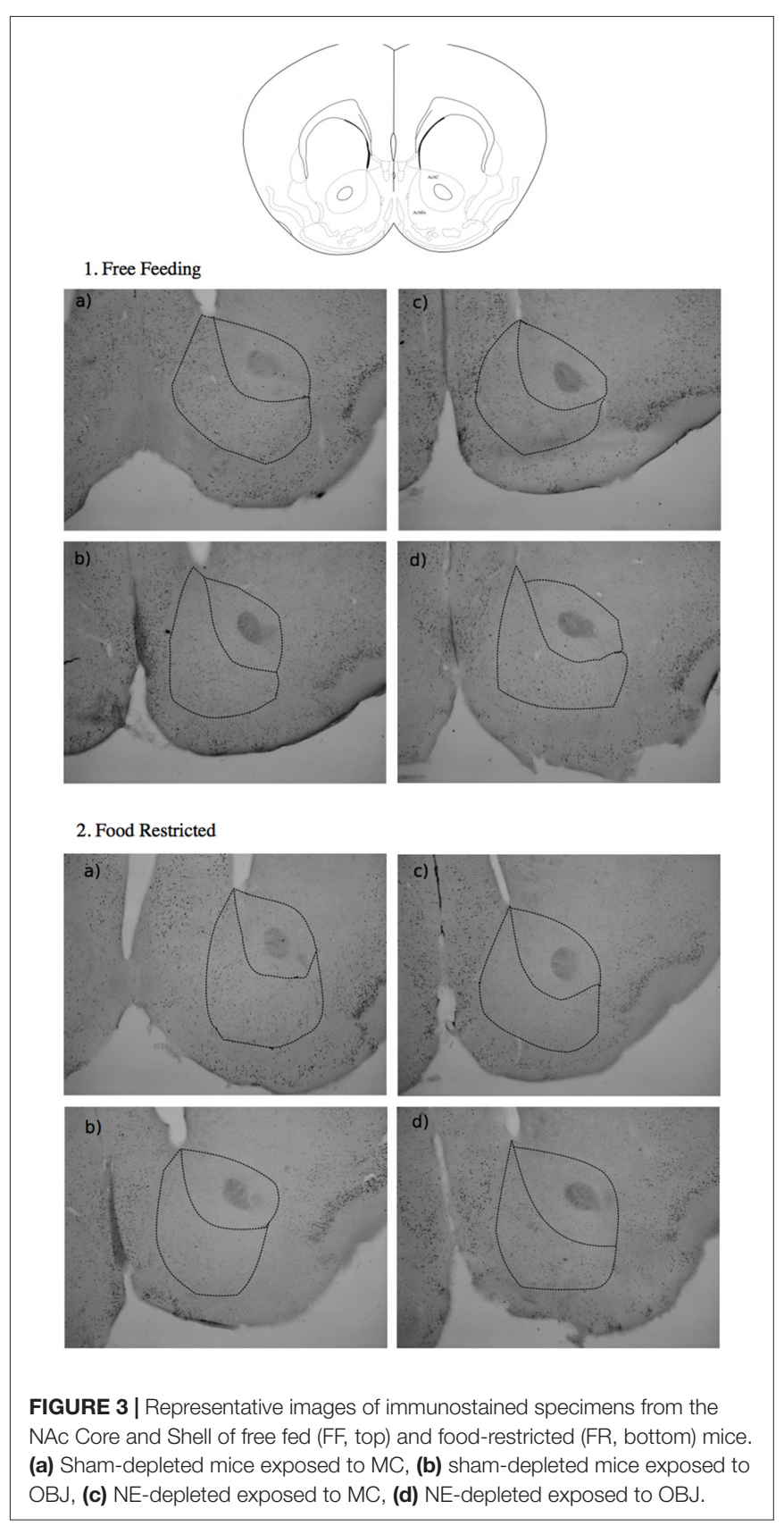

of FR but not FF mice. It is worth pointing out the discrepancy between present and previous results obtained in rats (Bassareo and Di Chiara, 1999), that can be easily explained by species difference as well as by differences in the type of milk chocolate used (white chocolate in the previous study: see Ventura et al., 2008 for details).

Our data also demonstrate that mesoaccumbens DA response to the novel palatable food by $\mathrm{FR}$ mice requires intact frontal cortical noradrenergic transmission because it was abolished by a selective depletion of frontal cortical NE. The noradrenergic depletion did not influence DA outflow in the NAc of FF mice although it has been shown to prevent the moderate increase of mpFC NE outflow elicited 

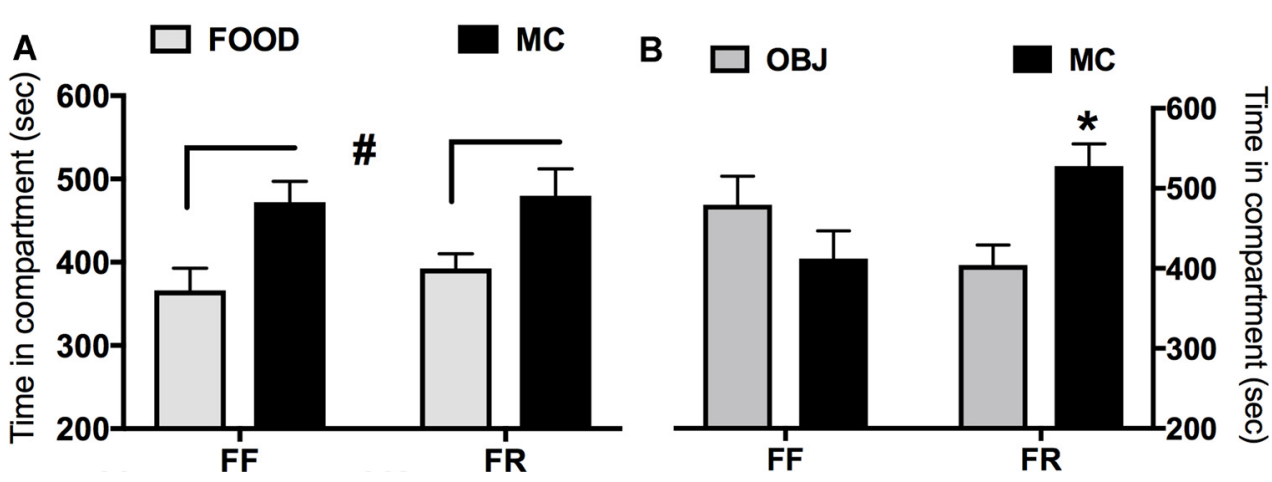

FIGURE 4 | Effects of restricted feeding (FR) on conditioned preference (seconds spent in compartment \pm SEM) for a context paired with milk chocolate (MC) in different experimental conditions. (A) Preference for the MC-paired compartment vs. the compartment paired with habitual lab chow (FOOD); (B) preference for MC-paired compartment vs. the compartment paired with novel inedible objects (OBJ). \#Main effect of the compartment ( $p<0.05$; see text for details). *Significantly different $(p<0.05)$ from time spent in the alternative compartment.

by MC in these mice (Ventura et al., 2008). This finding offers strong support to the view that DA outflow in NAc Shell is only controlled by large NE concentrations in $\mathrm{mpFC}$.

There was no effect of mpFC NE depletion on the amount of consumed chocolate although FR mice ate significantly more MC than FF mice (see "Materials and Methods" section), these data are in line with those obtained in mice exposed to the palatable food for a much longer time (Ventura et al., 2008) and with the general observation that feeding behavior does not require enhanced mesoaccumbens DA transmission (Nicola, 2016; Boekhoudt et al., 2017).

\section{A First Experience of MC Promotes a Different Pattern of c-fos Expression in the Striatum of ad libitum Fed and Food-Restricted Mice and Frontal Cortical NE Depletion Only Influences c-fos Expression Elicited by Incentive Stimuli in Food Restricted Mice}

A second set of experiments evaluated whether a first experience with MC engages different brain circuits depending on the feeding state of the organism. To this aim we evaluated the pattern of brain c-fos activation elicited by the palatable food because increasing evidence supports the use of this brain mapping strategy in rodents (Knapska et al., 2007; Ago et al., 2015; Jiménez-Sánchez et al., 2016). To control for the effect of stimulus novelty, known to activate c-fos expression in the brain (Jenkins et al., 2004; Struthers et al., 2005; Knapska et al., 2007; Rinaldi et al., 2010), we used exposure to a novel inedible object (OBJ).

The results obtained offer strong support to the tested hypothesis. Thus, only in FR mice NAc c-fos expression promoted by $\mathrm{MC}$ was larger than that promoted by $\mathrm{OBJ}$; moreover in these mice, but not in ad-libitum fed mice, mpFC $\mathrm{NE}$ depletion selectively reduced c-fos expression elicited by
$\mathrm{MC}$ in the NAc Shell, indicating the requirement of intact mpFC NE transmission. These findings parallel the results obtained with microdialysis and support a causal relationship between the two because of strong evidence for a major role of stimulation of DA receptors in striatal c-fos expression (Badiani et al., 1998; Barrot et al., 2000; Carr et al., 2003; BertranGonzalez et al., 2008; Colelli et al., 2010; Ago et al., 2015). By contrast, a larger increase of c-fos expression in OBJ- vs. MC-exposed mice was observed in the DMS of Sham-depleted mice. A strong activation elicited by the novel inedible object in the DMS is coherent with previous findings in mice and rats (Struthers et al., 2005; Rinaldi et al., 2010) and with the main role of DMS functioning for exploration of novel objects (Durieux et al., 2012). Restricted feeding reduced OBJ-induced c-fos expression in DMS and mpFC NE depletion abolished the effect of food restriction, suggesting an inhibitory control of frontal cortical NE on the induction of $\mathrm{c}$-fos expression in the DMS of FR mice. Moreover, although the first MC experience elicited a larger c-fos expression than OBJ in the NAc Core of FR mice, mpFC-NE depletion eliminated this difference by increasing $\mathrm{c}$-fos expression in $\mathrm{OBJ}$-exposed mice rather than by reducing $\mathrm{c}$-fos expression in $\mathrm{MC}$-exposed mice. Together, these findings support the hypothesis that in FR mice increased frontal cortical NE transmission enhances c-fos expression promoted by exploration of MC in the NAc Shell and inhibits c-fos expression induced by exploration of a novel inedible object in both the DMS and the NAc Core.

On the other hand, both FF and FR mice showed a larger increase of $\mathrm{c}$-fos expression in the CeA when exposed to $\mathrm{MC}$ than when exposed to $\mathrm{OBJ}$, and in both groups the response was still evident following mpFC NE depletion. The latter finding is in line with the view that induction of $\mathrm{c}$-fos expression in the CeA by novel palatable tastes is mediated by gustatory afferent information from the parabrachial nuclei of the pons (Koh et al., 2003; Knapska et al., 2007). Although CeA activation by novel tastes has been proposed to mediate food neophobia: an aversive response, this interpretation has been challenged by results of lesion 
studies (Reilly and Bornovalova, 2005) and by the observation that stimulation of $\mathrm{CeA} \mu$-opioid receptors enhances incentive salience of different stimuli including palatable food (Mahler and Berridge, 2012). Moreover, there is consistent evidence for a role of the CeA in the Pavlovian appetitive conditioning and, in particular, in place conditioning (Knapska et al., 2007; Rezayof et al., 2007). Therefore, activation of CeA could contribute to mpFC NE-independent MC-induced CPP in FF mice (Ventura et al., 2008).

\section{Only FR Mice Develop Conditioned Preference for a Context Paired with a Novel Palatable Food When the Other Is Associated with an Inedible Novel Object}

In FF mice there was no difference in NAc c-fos expression elicited by $\mathrm{MC}$ or $\mathrm{OBJ}$. The most conservative interpretation of this finding is that the two stimuli were equally salient possibly because of their novelty. Indeed, novel objects are strong incentive for rodents (Reichel and Bevins, 2008). This interpretation could also explain why both FF and FR mice develop conditioned preference for an MC-paired context when the other is associated with habitual lab chow although only in FR mice this conditioning is prevented by mpFC NE depletion (Ventura et al., 2008). In other words, motivational salience of MC could depend on novelty in FF but not in FR mice. To test this hypothesis, we trained FF and FR mice in an apparatus that contrasted a compartment associated with the novel palatable food with one associated with novel objects. We reasoned that if novelty motivates conditioned preference for the MC paired context in FF mice no preference should be observable when a different novel stimulus is associated with the other compartment.

The results obtained strongly supported this hypothesis. Indeed, FF mice did not develop conditioned preference for the compartment associated with MC when the other was associated with object novelty although, as previously reported (Ventura et al., 2008), they showed conditioned preference for the MC-paired compartment when the other was associated with a well-known taste. By contrast, FR mice preferred the MC-associated compartment in both experimental settings supporting the conclusion that incentive salience of $\mathrm{MC}$ and $\mathrm{MC}$-associated stimuli for these mice is unrelated to novelty. This conclusion supports the role of CeA in CPP induced by $\mathrm{MC}$ in FF but not in FR mice. Therefore, behavioral and $\mathrm{c}$-fos findings of the present experiments converge to indicate that different brain circuits process motivational salience of the novel palatable food in the two feeding conditions.

Finally, the observation that $\mathrm{OBJ}$ competes with $\mathrm{MC}$ for place conditioning in FF but not in FR mice indicates that the motivational salience of the novel palatable food is higher in the latter group. Indeed, a previous study reported that novel objects compete with low but not with high doses of cocaine for place conditioning (Reichel and Bevins, 2010). Moreover, because the first experience of MC prompts an increase on frontal cortical NE larger in FR then in FF mice (Ventura et al., 2008) these findings support the hypothesis that the extent of frontal cortical NE release elicited by an incentive stimulus is dependent on strength of its motivational salience (Puglisi-Allegra and Ventura, 2012).

\section{GENERAL CONCLUSION AND IMPLICATIONS}

The findings of the present study support the general conclusion that a specific brain circuit involving the NAc Shell through high NE levels in mpFC is engaged by addictive drugs, stress, and by palatable food in food restricted mice. Thus, as discussed, only blockade of alphal receptors, sensitive to high but not moderate frontal cortical NE concentrations (Ramos and Arnsten, 2007), prevents stress- (Nicniocaill and Gratton, 2007) and amphetamine-induced mesoaccumbens DA release (Darracq et al., 1998). Seemingly, only in FR mice, characterized by a significantly larger mpFC NE response MC than FF mice (Ventura et al., 2008), the palatable food enhances DA release and c-fos expression in the NAc Shell, and this effect is prevented by selective mpFC NE depletion.

The finding that in FR mice a novel palatable food engages a brain circuit engaged by addictive drugs and stress is not surprising. Indeed, food-restricted mice and rats show addiction-like behavioral and neural phenotypes in the laboratory (Cabib et al., 2000; Carr, 2011; Campus et al., 2017) and human data indicate that restrained eating is associated to loss of control, bingeing and counterproductive weight gain, whereas severe dieting is a risk factor for binge pathology and substance abuse (Carr, 2011). Therefore, the findings of the present study support the hypothesis that high frontal cortical concentration of $\mathrm{NE}$ can be responsible for dysfunctional motivation through engagement of a specific brain circuit.

Dysfunctional processing of motivationally salient stimuli has been proposed as trans-diagnostic phenotype of very different disturbances (Robinson and Berridge, 2001; Sinha and Jastreboff, 2013; Winton-Brown et al., 2014; Nusslock and Alloy, 2017), including schizophrenia (Kapur et al., 2005; Velligan et al., 2006; Reckless et al., 2015). The involvement of NE transmission in psychopathology has been long known and has supported development of pharmacological treatments aimed at adrenergic receptors (Ramos and Arnsten, 2007; Borodovitsyna et al., 2017; Maletic et al., 2017). The main target of these interventions is cognitive functioning (Arnsten, 2015), although there is also evidence that NE manipulation can affect the positive symptoms associated with schizophrenia (Borodovitsyna et al., 2017; Maletic et al., 2017). To these targets, present findings add dysfunctional motivation by supporting the involvement of high frontal cortical NE transmission in this trans-diagnostic phenotype (Robinson and Berridge, 2001; Kapur et al., 2005; Sinha and Jastreboff, 2013; Winton-Brown et al., 2014; Nusslock and Alloy, 2017). 


\section{AUTHOR CONTRIBUTIONS}

SC, ECL and SP-A planned the experiments and processed data; SC, ECL, SP-A and RV worked on the manuscript; ECL and RV performed experiments; $\mathrm{SC}$ wrote the manuscript.

\section{REFERENCES}

Abramoff, M. D., Magelhaes, P. J., and Ram, S. J. (2004). Image processing with ImageJ. Biophotonics Int. 11, 36-42.

Ago, Y., Hasebe, S., Nishiyama, S., Oka, S., Onaka, Y., Hashimoto, H., et al. (2015). The female encounter test: a novel method for evaluating reward-seeking behavior or motivation in mice. Int. J. Neuropsychopharmacol. 18:pyv062. doi: 10.1093/ijnp/pyv062

Arnsten, A. F. T. (2015). Stress weakens prefrontal networks: molecular insults to higher cognition. Nat. Neurosci. 18, 1376-1385. doi: 10.1038/nn.4087

Badiani, A., Oates, M. M., Day, H. E., Watson, S. J., Akil, H., and Robinson, T. E. (1998). Amphetamine-induced behavior, dopamine release, and c-fos mRNA expression: modulation by environmental novelty. J. Neurosci. 18, 10579-10593.

Baldo, B. A., Pratt, W. E., Will, M. J., Hanlon, E. C., Bakshi, V. P., and Cador, M. (2013). Principles of motivation revealed by the diverse functions of neuropharmacological and neuroanatomical substrates underlying feeding behavior. Neurosci. Biobehav. Rev. 37, 1985-1998. doi: 10.1016/j.neubiorev. 2013.02.017

Barrot, M., Marinelli, M., Abrous, D. N., Rougé-Pont, F., Le Moal, M., and Piazza, P. V. (2000). The dopaminergic hyper-responsiveness of the shell of the nucleus accumbens is hormone-dependent. Eur. J. Neurosci. 12, 973-979. doi: 10.1046/j.1460-9568.2000.00996.x

Bassareo, V., and Di Chiara, G. (1999). Modulation of feeding-induced activation of mesolimbic dopamine transmission by appetitive stimuli and its relation to motivational state. Eur. J. Neurosci. 11, 4389-4397. doi: 10.1046/j.1460-9568. 1999.00843.x

Bechara, A., and van der Kooy, D. (1992). A single brain stem substrate mediates the motivational effects of both opiates and food in nondeprived rats but not in deprived rats. Behav. Neurosci. 106, 351-363. doi: 10.1037/0735-7044. 106.2.351

Berridge, K. C., and Kringelbach, M. L. (2015). Pleasure systems in the brain. Neuron 86, 646-664. doi: 10.1016/j.neuron.2015.02.018

Bertran-Gonzalez, J., Bosch, C., Maroteaux, M., Matamales, M., Hervé, D., Valjent, E., et al. (2008). Opposing patterns of signaling activation in Dopamine D1 and D2 receptor-expressing striatal neurons in response to cocaine and haloperidol. J. Neurosci. 28, 5671-5685. doi: 10.1523/JNEUROSCI.1039-08. 2008

Bimpisidis, Z., De Luca, M. A., Pisanu, A., and Di Chiara, G. (2013). Lesion of medial prefrontal dopamine terminals abolishes habituation of accumbens shell dopamine responsiveness to taste stimuli. Eur. J. Neurosci. 37, 613-622. doi: 10.1111/ejn.12068

Boekhoudt, L., Roelofs, T. J. M., de Jong, J. W., de Leeuw, A. E., Luijendijk, M. C. M., Wolterink-Donselaar, I. G., et al. (2017). Does activation of midbrain dopamine neurons promote or reduce feeding? Int. J. Obes. 41, 1131-1140. doi: 10.1038/ijo.2017.74

Borodovitsyna, O., Flamini, M., and Chandler, D. (2017). Noradrenergic modulation of cognition in health and disease. Neural Plast. 2017:6031478. doi: $10.1155 / 2017 / 6031478$

Cabib, S., Orsini, C., Le Moal, M., and Piazza, P. V. (2000). Abolition and reversal of strain differences in behavioral responses to drugs of abuse after a brief experience. Science 289, 463-465. doi: 10.1126/science.289. 5478.463

Cabib, S., and Puglisi-Allegra, S. (2012). The mesoaccumbens dopamine in coping with stress. Neurosci. Biobehav. Rev. 36, 79-89. doi: 10.1016/j.neubiorev.2011. 04.012

Campus, P., Canterini, S., Orsini, C., Fiorenza, M. T., Puglisi-Allegra, S., and Cabib, S. (2017). Stress-induced reduction of dorsal striatal D2 dopamine receptors prevents retention of a newly acquired adaptive coping strategy. Front. Pharmacol. 8:621. doi: 10.3389/fphar.2017.00621

\section{FUNDING}

This research was funded by Research Projects of Sapienza University of Rome grant no. ATENEO AA 2016.

Carr, K. D. (2011). Food scarcity, neuroadaptations, and the pathogenic potential of dieting in an unnatural ecology: binge eating and drug abuse. Physiol. Behav. 104, 162-167. doi: 10.1016/j.physbeh.2011.04.023

Carr, K. D., Tsimberg, Y., Berman, Y., and Yamamoto, N. (2003). Evidence of increased dopamine receptor signaling in food-restricted rats. Neuroscience 119, 1157-1167. doi: 10.1016/s0306-4522(03)00227-6

Colelli, V., Campus, P., Conversi, D., Orsini, C., and Cabib, S. (2014). Either the dorsal hippocampus or the dorsolateral striatum is selectively involved in consolidation of forced swim-induced immobility depending on genetic background. Neurobiol. Learn. Mem. 111, 49-55. doi: 10.1016/j.nlm.2014. 03.004

Colelli, V., Fiorenza, M. T., Conversi, D., Orsini, C., and Cabib, S. (2010). Strainspecific proportion of the two isoforms of the dopamine D2 receptor in the mouse striatum: associated neural and behavioral phenotypes. Genes Brain Behav. 9, 703-711. doi: 10.1111/j.1601-183X.2010.00604.x

Conversi, D., Bonito-Oliva, A., Orsini, C., and Cabib, S. (2006). Habituation to the test cage influences amphetamine-induced locomotion and Fos expression and increases FosB/ $\Delta$ FosB-like immunoreactivity in mice. Neuroscience 141 , 597-605. doi: 10.1016/j.neuroscience.2006.04.003

Conversi, D., Orsini, C., and Cabib, S. (2004). Distinct patterns of Fos expression induced by systemic amphetamine in the striatal complex of C57BL/6JICo and DBA/2JICo inbred strains of mice. Brain Res. 1025, 59-66. doi: 10.1016/j. brainres.2004.07.072

Darracq, L., Blanc, G., Glowinski, J., and Tassin, J. P. (1998). Importance of the noradrenaline-dopamine coupling in the locomotor activating effects of D-amphetamine. J. Neurosci. 18, 2729-2739.

Deutch, A. Y., Clark, W. A., and Roth, R. H. (1990). Prefrontal cortical dopamine depletion enhances the responsiveness of mesolimbic dopamine neurons to stress. Brain Res. 521, 311-315. doi: 10.1016/0006-8993(90) 91557-w

Di Chiara, G., and Bassareo, V. (2007). Reward system and addiction: what dopamine does and doesn't do. Curr. Opin. Pharmacol. 7, 69-76. doi: 10.1016/j. coph.2006.11.003

Doherty, M. D., and Gratton, A. (1996). Medial prefrontal cortical D1 receptor modulation of the meso-accumbens dopamine response to stress: an electrochemical study in freely-behaving rats. Brain Res. 715, 86-97. doi: 10.1016/0006-8993(95)01557-4

Durieux, P. F., Schiffmann, S. N., and de Kerchove d'Exaerde, A. (2012). Differential regulation of motor control and response to dopaminergic drugs by $\mathrm{D} 1 \mathrm{R}$ and $\mathrm{D} 2 \mathrm{R}$ neurons in distinct dorsal striatum subregions. EMBO J. 31, 640-653. doi: 10.1038/emboj.2011.400

Faure, A., Reynolds, S. M., Richard, J. M., and Berridge, K. C. (2008). Mesolimbic dopamine in desire and dread: enabling motivation to be generated by localized glutamate disruptions in nucleus accumbens. J. Neurosci. 28, 7184-7192. doi: 10.1523/JNEUROSCI.4961-07.2008

Fields, H. L., and Margolis, E. B. (2015). Understanding opioid reward. Trends Neurosci. 38, 217-225. doi: 10.1016/j.tins.2015.01.002

Fiore, V. G., Mannella, F., Mirolli, M., Latagliata, E. C., Valzania, A., Cabib, S., et al. (2015). Corticolimbic catecholamines in stress: a computational model of the appraisal of controllability. Brain Struct. Funct. 220, 1339-1353. doi: 10.1007/s00429-014-0727-7

Franklin, K. B. J., and Paxinos, G. (2001). The Mouse Brain in Stereotaxic Coordinates. San Diego, CA: Academic Press.

Jenkins, T. A., Amin, E., Pearce, J. M., Brown, M. W., and Aggleton, J. P. (2004). Novel spatial arrangements of familiar visual stimuli promote activity in the rat hippocampal formation but not the parahippocampal cortices: a c-fos expression study. Neuroscience 124, 43-52. doi: 10.1016/j.neuroscience.2003. 11.024

Jiménez-Sánchez, L., Castañé, A., Pérez-Caballero, L., Grifoll-Escoda, M., LöpezGil, X., Campa, L., et al. (2016). Activation of AMPA receptors mediates the 
antidepressant action of deep brain stimulation of the infralimbic prefrontal cortex. Cereb. Cortex 26, 2778-2789. doi: 10.1093/cercor/bhv133

Kapur, S., Mizrahi, R., and Li, M. (2005). From dopamine to salience to psychosis-linking biology, pharmacology and phenomenology of psychosis. Schizophr. Res. 79, 59-68. doi: 10.1016/j.schres.2005.01.003

Knapska, E., Radwanska, K., Werka, T., and Kaczmarek, L. (2007). Functional internal complexity of amygdala: focus on gene activity mapping after behavioral training and drugs of abuse. Physiol. Rev. 87, 1113-1173. doi: 10.1152/physrev.00037.2006

Koh, M. T., Wilkins, E. E., and Bernstein, I. L. (2003). Novel tastes elevate c-fos expression in the central amygdala and insular cortex: implication for taste aversion learning. Behav. Neurosci. 117, 1416-1422. doi: 10.1037/0735-7044. 117.6.1416

Mahler, S. V., and Berridge, K. C. (2012). What and when to "want"? Amygdalabased focusing of incentive salience upon sugar and sex. Psychopharmacology 221, 407-426. doi: 10.1007/s00213-011-2588-6

Maletic, V., Eramo, A., Gwin, K., Offord, S. J., and Duffy, R. A. (2017). The role of norepinephrine and its $\alpha$-adrenergic receptors in the pathophysiology and treatment of major depressive disorder and schizophrenia: a systematic review. Front. Psychiatry 8:42. doi: 10.3389/fpsyt.2017.00042

Nader, K., Bechara, A., and van der Kooy, D. (1997). Neurobiological constraints on behavioral models of motivation. Annu. Rev. Psychol. 48, 85-114. doi: 10.1146/annurev.psych.48.1.85

Nicniocaill, B., and Gratton, A. (2007). Medial prefrontal cortical a1 adrenoreceptor modulation of the nucleus accumbens dopamine response to stress in Long-Evans rats. Psychopharmacology 191, 835-842. doi: 10.1007/s00213-007-0723-1

Nicola, S. M. (2016). Reassessing wanting and liking in the study of mesolimbic influence on food intake. Am. J. Physiol. Regul. Integr. Comp. Physiol. 311, R811-R840. doi: 10.1152/ajpregu.00234.2016

Nusslock, R., and Alloy, L. B. (2017). Reward processing and mood-related symptoms: an RDoC and translational neuroscience perspective. J. Affect. Disord. 216, 3-16. doi: 10.1016/j.jad.2017.02.001

Paolone, G., Conversi, D., Caprioli, D., Bianco, P. D., Nencini, P., Cabib, S., et al. (2007). Modulatory effect of environmental context and drug history on heroin-induced psychomotor activity and fos protein expression in the rat brain. Neuropsychopharmacology 32, 2611-2623. doi: 10.1038/sj.npp.1301388

Pascucci, T., Ventura, R., Latagliata, E. C., Cabib, S., and Puglisi-Allegra, S. (2007). The medial prefrontal cortex determines the accumbens dopamine response to stress through the opposing influences of norepinephrine and dopamine. Cereb. Cortex 17, 2796-2804. doi: 10.1093/cercor/bhm008

Puglisi-Allegra, S., and Ventura, R. (2012). Prefrontal/accumbal catecholamine system processes high motivational salience. Front. Behav. Neurosci. 6:31. doi: 10.3389/fnbeh.2012.00031

Pujara, M. S., Philippi, C. L., Motzkin, J. C., Baskaya, M. K., and Koenigs, M. (2016). Ventromedial prefrontal cortex damage is associated with decreased ventral striatum volume and response to reward. J. Neurosci. 36, 5047-5054. doi: 10.1523/JNEUROSCI.4236-15.2016

Quiroz, C., Orrú, M., Rea, W., Ciudad-Roberts, A., Yepes, G., Britt, J. P., et al. (2016). Local control of extracellular dopamine levels in the medial nucleus accumbens by a glutamatergic projection from the infralimbic cortex. J. Neurosci. 36, 851-859. doi: 10.1523/JNEUROSCI.2850-15.2016

Ramos, B. P., and Arnsten, A. F. (2007). Adrenergic pharmacology and cognition: focus on the prefrontal cortex. Pharmacol. Ther. 113, 523-536. doi: 10.1016/j. pharmthera.2006.11.006

Reckless, G. E., Andreassen, O. A., Server, A., Østefjells, T., and Jensen, J. (2015). Negative symptoms in schizophrenia are associated with aberrant striato-cortical connectivity in a rewarded perceptual decision-making task. Neuroimage Clin. 8, 290-297. doi: 10.1016/j.nicl.2015.04.025

Reichel, C. M., and Bevins, R. A. (2008). Competition between the conditioned rewarding effects of cocaine and novelty. Behav. Neurosci. 122, 140-150. doi: $10.1037 / 0735-7044.122 .1 .140$

Reichel, C. M., and Bevins, R. A. (2010). Competition between novelty and cocaine conditioned reward is sensitive to drug dose and retention interval. Behav. Neurosci. 124, 141-151. doi: 10.1037/a0018226
Reilly, S., and Bornovalova, M. A. (2005). Conditioned taste aversion and amygdala lesions in the rat: a critical review. Neurosci. Biobehav. Rev. 29, 1067-1088. doi: 10.1016/j.neubiorev.2005.03.025

Rezayof, A., Golhasani-Keshtan, F., Haeri-Rohani, A., and Zarrindast, M. R. (2007). Morphine-induced place preference: involvement of the central amygdala NMDA receptors. Brain Res. 1133, 34-41. doi: 10.1016/j.brainres. 2006.11.049

Richard, J. M., and Berridge, K. C. (2013). Prefrontal cortex modulates desire and dread generated by nucleus accumbens glutamate disruption. Biol. Psychiatry 73, 360-370. doi: 10.1016/j.biopsych.2012.08.009

Richard, J. M., Plawecki, A. M., and Berridge, K. C. (2013). Nucleus accumbens GABAergic inhibition generates intense eating and fear that resists environmental retuning and needs no local dopamine. Eur. J. Neurosci. 37, 1789-1802. doi: 10.1111/ejn.12194

Rinaldi, A., Romeo, S., Agustín-Pavón, C., Oliverio, A., and Mele, A. (2010). Distinct patterns of Fos immunoreactivity in striatum and hippocampus induced by different kinds of novelty in mice. Neurobiol. Learn. Mem. 94, 373-381. doi: 10.1016/j.nlm.2010.08.004

Robinson, T. E., and Berridge, K. C. (2001). Incentive-sensitization and addiction. Addiction 96, 103-114. doi: 10.1046/j.1360-0443.2001.96 11038.x

Sinha, R., and Jastreboff, A. M. (2013). Stress as a common risk factor for obesity and addiction. Biol. Psychiatry 73, 827-835. doi: 10.1016/j.biopsych.2013. 01.032

Struthers, W. M., DuPriest, A., and Runyan, J. (2005). Habituation reduces novelty-induced FOS expression in the striatum and cingulate cortex. Exp. Brain Res. 167, 136-140. doi: 10.1007/s00221-005-0061-7

Velligan, D. I., Kern, R. S., and Gold, J. M. (2006). Cognitive rehabilitation for schizophrenia and the putative role of motivation and expectancies. Schizophr. Bull. 32, 474-485. doi: 10.1093/schbul/sbj071

Ventura, R., Alcaro, A., and Puglisi-Allegra, S. (2005). Prefrontal cortical norepinephrine release is critical for morphine-induced reward, reinstatement and dopamine release in the nucleus accumbens. Cereb. Cortex 15, 1877-1886. doi: 10.1093/cercor/bhi066

Ventura, R., Cabib, S., Alcaro, A., Orsini, C., and Puglisi-Allegra, S. (2003). Norepinephrine in the prefrontal cortex is critical for amphetamine-induced reward and mesoaccumbens dopamine release. J. Neurosci. 23, 1879-1885.

Ventura, R., Latagliata, E. C., Morrone, C., La Mela, I., and PuglisiAllegra, S. (2008). Prefrontal norepinephrine determines attribution of "high" motivational salience. PLoS One 3:e3044. doi: 10.1371/journal.pone. 0003044

Ventura, R., Morrone, C., and Puglisi-Allegra, S. (2007). Prefrontal/accumbal catecholamine system determines motivational salience attribution to both reward- and aversion-related stimuli. Proc. Natl. Acad. Sci. U S A 104, 5181-5186. doi: 10.1073/pnas.0610178104

Ventura, R., and Puglisi-Allegra, S. (2005). Environment makes amphetamineinduced dopamine release in the nucleus accumbens totally impulsedependent. Synapse 58, 211-214. doi: 10.1002/syn.20197

Winton-Brown, T. T., Fusar-Poli, P., Ungless, M. A., and Howes, O. D. (2014). Dopaminergic basis of salience dysregulation in psychosis. Trends Neurosci. 37, 85-94. doi: 10.1016/j.tins.2013.11.003

Conflict of Interest Statement: The authors declare that the research was conducted in the absence of any commercial or financial relationships that could be construed as a potential conflict of interest.

The reviewer LP and handling Editor declared their shared affiliation.

Copyright (c) 2018 Latagliata, Puglisi-Allegra, Ventura and Cabib. This is an open-access article distributed under the terms of the Creative Commons Attribution License (CC BY). The use, distribution or reproduction in other forums is permitted, provided the original author(s) and the copyright owner are credited and that the original publication in this journal is cited, in accordance with accepted academic practice. No use, distribution or reproduction is permitted which does not comply with these terms. 